\title{
Sulla regolarizzazione delle soluzioni del problema di Cauchy-Dirichlet per un'equazione parabolica di ordine superiore.
}

\author{
Giuseppe ARNese (a Bari) (*) (**)
}

Sunto. - Si studia il problema non omogeneo di Cauchy-Dirichlet per un'equazione parabolica di ordine superiore, in forma variazionale, provando un teorema di esistenza ed unicità ed una maggiorazione analoga a quella contenuta nel teorema del massimo modulo stabilito da C. Miranda per le equazioni ellittiche di ordine superiore.

In alcuni miei lavori [2], [3] ho studiato il problema di DIRIoHtet per una equazione parabolica di ordine $2 m$ in $n$ variabili in un cilindro, precisamente il problema:

$$
\begin{aligned}
& \iint_{\Omega}\left\{\sum_{|p| \cdot|g| \leqslant m}(-1)^{|p|} a_{p q} D^{p} u D^{q} \bar{\tau}-u \frac{\partial \bar{\tau}}{\partial t}\right\} d x d t=\iint_{\Omega} \sum_{|\alpha| \leqslant m} f_{\alpha} \cdot D^{\alpha} \bar{\tau} d x d t \\
& \quad(\forall \tau \in \mathfrak{D}(\Omega) ; \Omega=G \times] 0, T\left[, G \subset \boldsymbol{R}^{n}\right) \\
& \begin{array}{l}
\frac{d^{j-1} u}{d \nu^{j-1}}\left(x^{\prime}, t\right)=\varphi_{j}\left(x^{\prime}, t\right) \quad\left(j=1, \ldots, m ;\left(x^{\prime}, t\right) \in \partial G \times[0, T]\right) \\
u(x, 0)=\psi(x) \quad(x \in \bar{G})
\end{array}
\end{aligned}
$$

ove $f=\left(f_{\alpha}\right)_{|\alpha| \leqslant m}$ è un sistema di funzioni tali che:

$$
M_{\lambda}(f)=\sum_{|\alpha| \leqslant m} \sup _{(x, t) \in \Omega} d(x)^{m-|\alpha|+1-\lambda}\left|f_{\alpha}(x, t)\right|<+\infty \quad\left(\frac{1}{2}<\lambda<1\right)
$$

$d(x)$ essendo la distanza di $(x, t)$ da $\partial G \times[0, T]$.

In [2] ho dimostrato un teorema di esistenza ed unicità per il problema omogeneo $\left(\varphi_{j}=0\right.$ per $\left.j=1, \ldots, m ; \psi=0\right)$ stabilendo, altresì, una formula di maggiorazione che può ritenersi l'estensione alle equazioni paraboliche di ordine $2 m$ del teorema di massimo modulo stabilito da C. MrRaNDa [9] per il problema di DrRIoHLET, anche non omogeneo, relativo alle equazioni ellittiche di ordine $2 \mathrm{~m}$.

In [3] ho stabilito risultati analoghi per il problema non omogeneo ma soltanto in casi particolari a causa della difficoltà di costruire, nel caso generale, una fun-

$\left(^{*}\right)$ Entrato in Redazione il 12 luglio 1971.

(**) Lavoro eseguito nell'ambito dell'attivita dei gruppi di ricerca del C.N.R.

2- Annali ai Matematica 
zione verificante le condizioni al contorno deI problema (I), con singolarità non troppo elevate sulla frontiera laterale di $\Omega$, mediante la quale poter ricondurre il problema non omogeneo a quello omogeneo.

In questo mio lavoro, studio ancora il problema non omogeneo (I) avvalendomi, fra l'altro, di alcuni risultati, da me stabiliti in un recente lavoro [4] dedicato allo studio di certi potenziali relativi ad equazioni paraboliche di ordine $2 m$, che permettono la costruzione della suddetta funzione ausiliaria.

Nel presente lavoro, inoltre, sostituisco la (II) con la seguente:

$$
M_{\lambda}(f)=\sum_{|\alpha| \leqslant m} \sup _{(x, t) \in \Omega}\left[\inf \left(d(x), t^{1 / 2 m}\right)\right]^{m-\mid x_{i}+1-\lambda}\left|f_{\alpha}(x, t)\right|<+\infty \quad\left(\frac{1}{2}<\lambda<1\right)
$$

consentendo, quindi, alle funzioni $f_{\alpha}(|\alpha| \leqslant m)$ di avere delle singolarità anche sulla base inferiore di $\Omega$. Questa generalizzazione sembra naturale, trattandosi di un problema parabolico, ma ha richiesto il superamento di non lievi difficoltà. In particolare è stato necessario perfezionare i risultati relativi al caso omogeneo, utilizzando spazi di funzioni con derivata frazionaria rispetto a $t$ (Teor. 3.2).

Dimostro, infine, che supposta verificata la (III) e in convenienti ipotesi sui coefficienti e sui dati al contorno, il problema (I) ammette un'unica soluzione $u \in C_{x, t}^{m-1+\lambda, \lambda / 2 m}(\bar{\Omega}) \cap_{R} W_{2}^{m, \frac{1}{2}}(\Omega)$ per la quale sussiste la maggiorazione:

$$
\begin{aligned}
& \sum_{|\alpha| \leqslant m-1} \sup _{\bar{\Omega}}\left|D^{\alpha} u(x, t)\right|+\sum_{|\alpha|=m-1} \sup _{\bar{\Omega}} \frac{\left|D^{\alpha} u\left(x^{\prime}, t\right)-D^{\alpha} u\left(x^{\prime \prime}, t\right)\right|}{\left|x^{\prime}-x^{\prime \prime}\right|^{\lambda}}+ \\
& +\sum_{|x| \leqslant m-1} \sup _{\bar{\Omega}} \frac{\left|D^{\alpha} u\left(x, t^{\prime}\right)-D^{\alpha} u\left(x, t^{\prime \prime}\right)\right|}{\left|t^{\prime}-t^{\prime \prime}\right|^{(m-1-|x|+\lambda) / 2 m}}+\sum_{|\alpha| \leqslant m}\left|D^{\alpha} u\right|_{L^{2}(\Omega)}+\inf \left(\int_{\sigma} d x \int_{-\infty}^{+\infty}|t||\mathcal{F}(P u)|^{2} d t\right)^{\frac{1}{2}} \leqslant \\
& \left.\leqslant c\left(M_{\lambda}(f)+\sum_{j=1}^{m} \mid \varphi_{j}, \partial G \times[0, T]\right]_{m-j, 0}^{(\lambda, 2 m)}+|\psi, \bar{G}|_{m-1}^{(\lambda)}\right)
\end{aligned}
$$

ove l'estremo inferiore è preso sui prolungamenti di $u$ in $G \times]-\infty,+\infty[\mathrm{e}$, a secondo membro, oltre $M_{\lambda}(f)$, compaiono certe norme hölderiane dei dati sulla frontiera (Teor. 3.4).

Nel n. 1 di questo lavoro sono raccolte le notazioni usate; il n. 2 è dedicato a dei lemmi concernenti i potenziali e che permettono la costruzione della funzione ausiliaria; nel n. 3, dopo il fondamentale Lemma 3.1, vi sono i risultati principali. di questo lavoro (Teor. 3.2 e Teor. 3.4).

\section{1. - Notazioni concernenti spazi funzionali.}

Denotiamo con $\boldsymbol{R}^{n}$ lo spazio euclideo ad $n$ dimensioni, con $x=\left(x_{1}, \ldots, x_{n}\right)$ un suo punto, con $(x, t)=\left(x_{1}, \ldots, x_{n}, t\right)=\left(x^{\prime}, x_{n}, t\right)$ un punto di $\boldsymbol{R}^{n+1}$.

Come di consueto, poniamo anche:

$$
\begin{aligned}
& \alpha=\left(\alpha_{1}, \ldots, \alpha_{n}\right) \quad\left(\alpha_{i} \text { intero } \geqslant 0 \forall i=1, \ldots, n\right) \\
& |\alpha|=\sum_{i=1}^{n} \alpha_{i}, \quad D^{\alpha}=\frac{\partial|\alpha|}{\partial x_{1}^{\alpha_{1}} \ldots \partial x_{n}^{\alpha_{n}}} .
\end{aligned}
$$


Richiamiamo, brevemente, definizioni e notazioni concernenti aleuni spazi funzionali.

Se $A$ è un dominio di $\boldsymbol{R}^{n}$, con $C^{k}(A)(k \in N)$ denotiamo lo spazio delle funzioni complesse $\phi$ definite in $A$, ivi continue e limitate con le loro derivate di ordine minore o eguale a $k$, normato ponendo:

$$
|\varphi, A|_{k}=\sum_{|\alpha| \leqslant k} \sup _{x \in A}\left|D^{\alpha} \varphi(x)\right|
$$

Con $C^{k+\lambda}(A)(k \in N, 0<\lambda<1)$ denotiamo lo spazio delle funzioni $\varphi \in C^{k}(A)$ tali che:

$$
\left.\sup _{x^{\prime}, x^{\prime \prime} \in A} \frac{\left|D^{\alpha} \varphi\left(x^{\prime}\right)-D^{\alpha} \varphi\left(x^{\prime \prime}\right)\right|}{\left|x^{\prime}-x^{\prime \prime}\right|^{\lambda}}<+\infty \quad \quad \text { (per }|\alpha|=k\right)
$$

normato ponendo:

$$
|\varphi, A|_{k}^{(\alpha)}=|\varphi, A|_{k}+\sum_{|x|=k} \sup _{x^{\prime}, x^{\prime} \in A} \frac{\left|D^{\alpha} \varphi\left(x^{\prime}\right)-D^{\alpha} \varphi\left(x^{\prime \prime}\right)\right|}{\left|x^{\prime}-x^{\prime \prime}\right|^{\lambda}} .
$$

Se $G$ è un aperto di $\boldsymbol{R}^{n}$ e se $T$ è un numero reale positivo, poniamo $\left.\Omega=G \times\right] 0, T[$.

Con $O_{s, t}^{k, 0}(\bar{\Omega})(k \in N)$ denotiamo lo spazio delle funzioni continue e limitate in $\bar{\Omega}$ con le derivate parziali rispetto ad $x$ fino all'ordine $k$; con $C_{x, t}^{k+\lambda, 0}(\bar{\Omega})(k \in N, 0<\lambda<1)$ denotiamo lo spazio delle funzioni $\varphi \in C_{x, t}^{x, 0}(\bar{\Omega})$ tali che:

$$
\left.\sup _{\left(x^{\prime}, t\right),\left(x^{\prime \prime}, t\right) \in \bar{\Omega}} \frac{\left|D^{\alpha} \varphi\left(x^{\prime}, t\right)-D^{\alpha} \varphi\left(x^{\prime \prime}, t\right)\right|}{\left|x^{\prime}-x^{\prime \prime}\right|^{\lambda}}<+\infty \quad \text { (per }|\alpha|=k\right) \text {. }
$$

Oon $C_{x, t}^{k+\lambda, \lambda / 2 m}(\bar{\Omega})(k \in N, 0<\lambda<1, m$ intero $\geqslant 1)$ designamo lo spazio delle funzioni $\varphi \in C_{x, t}^{k, 0}(\bar{\Omega})$ tali che risulti finita la norma:

$$
\begin{aligned}
& |\varphi, \bar{\Omega}|_{k, 0}^{(\lambda, 2 m)}=\sum_{|\alpha| \leqslant k} \sup _{(x, t) \in \bar{\Omega}}\left|D^{\alpha} \varphi(x, t)\right|+
\end{aligned}
$$

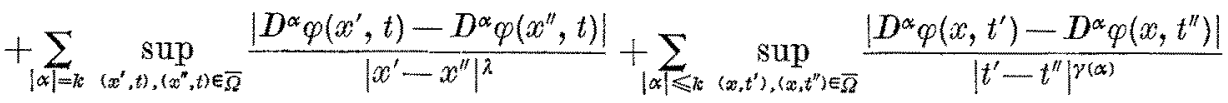

ove $\gamma(\alpha)=\inf (1,(k-|\alpha|+\lambda) / 2 m)$.

Con $C_{x, t}^{2 m, 1}(\Omega)$ denotiamo lo spazio delle funzioni continue in $\Omega$ con le derivate rispetto ad $x$ sino a quelle di ordine $2 m$ e con la derivata parziale prima rispetto a $t$.

Riportiamo, ora, alcune definizioni concernenti spazi di funzioni con derivata frazionaria rispetto a $t\left({ }^{1}\right)$.

Se $G$ è un insieme aperto limitato di $\boldsymbol{R}^{n}$ e $\left.Q=G \times\right]-\infty$, $+\infty[$, denotiamo con

(1) Cfr. [8]. 
$W_{2}^{m, \frac{1}{2}}(Q)$ lo spazio delle $\varphi \in L^{2}(Q)$ tali che:

$$
D^{\alpha} \varphi \in L^{2}(Q) \quad(|\alpha| \leqslant m) ; \quad|t|^{\frac{1}{2}} \cdot|\varsubsetneqq \varphi(\infty, t)| \in L^{2}(Q)
$$

ove le derivate sono intese nel senso delle distribuzioni e si è posto:

$$
\mathcal{F} \varphi(x, t)=\frac{1}{2 \pi} \int_{-\infty}^{+\infty} \exp [-i t \eta] \varphi(x, \eta) d \eta
$$

Fatte, inoltre, per $\varphi \in W_{a}^{m, \frac{1}{3}}(Q)$ le posizioni:

$$
\begin{aligned}
& \|\varphi\|_{0, \frac{1}{2}}=\frac{1}{2 \pi}\left(\iint_{Q}|t| \cdot|\mathscr{F} \varphi(x, t)|^{2} d x d t\right)^{\frac{1}{2}} \\
& \|\varphi, Q\|_{m, \frac{1}{1}}=\left(\sum_{|\alpha| \leqslant m}\left\|D^{\alpha} \varphi\right\|_{L^{2}(Q)}^{2}+\|\varphi\|_{0,1}^{2}\right)^{\frac{1}{2}}
\end{aligned}
$$

lo spazio $W_{2}^{m, \frac{1}{2}}(Q)$ viene munito della norma $\|\cdot, Q\|_{m . \frac{1}{2}}$.

Denotiamo con ${ }^{0} W_{2}^{m \cdot \frac{1}{2}}(Q)$ la chiusura di $\mathcal{D}(Q)$ nello spazio $W_{2}^{m, \frac{1}{2}}(Q)$.

Considerato l'insieme $\Omega=G \times] 0, T\left[(T>0)\right.$, designamo con ${ }_{n} W_{2}^{n, \frac{1}{2}}(\Omega)$ (rispett. ${ }_{R}^{0} W_{2}^{m, \frac{3}{3}}(\Omega)$ ) lo spazio delle funzioni $u \in L^{2}(\Omega)$ che ammettono un prolungamento $P u \in W_{2}^{m, \frac{1}{2}}(Q)$ (rispett. $\left.P u \in \in^{0} W_{2}^{m, \frac{1}{2}}(\Omega)\right)$ munito della norma:

$$
\|u, \Omega\|_{m, \underline{z}}=\inf _{P_{u \in X}}\|P u, Q\|_{m, \frac{1}{2}}
$$

ove $X$ è l'insieme di tutti i prolungamenti $P u \in W_{2}^{m, \frac{1}{2}}(Q)$ (rispett. $P u \in{ }^{0} W_{2}^{m, \mathbf{z}}(Q)$ ). Se $u \in{ }_{R}^{0} W_{2}^{m, \frac{1}{2}}(\Omega)$ e $\varphi \in \mathscr{D}(\Omega)$, poniamo:

$$
\begin{gathered}
\langle u, \varphi\rangle_{\Omega}=-\iint_{\Omega} \bar{u} \frac{\partial \varphi}{\partial t} d x d t \\
|u|_{\Omega}=\sup _{\varphi \in \Gamma}\left|\langle u, \varphi\rangle_{\Omega}\right|
\end{gathered}
$$

ove $Y$ è l'insieme delle $\varphi \in \mathscr{D}(\Omega)$ tali che $\|\varphi, \Omega\|_{m, \frac{1}{2}} \leqslant 1$. Si denota con ${ }_{s}^{0} W_{2}^{m, \frac{1}{2}}(\Omega)$ l'insieme delle funzioni $u \in_{n}^{0} W_{2}^{m, t}(\Omega)$ tali che $|u|_{\Omega}<+\infty$ e che ammettono un prolungamento $P u \in{ }^{0} W_{2}^{m, \frac{z}{z}}(Q)$ tale che $P u(x, t)=0$ per $t<0$; in tale spazio si introduce la norma:

$$
\|u, \Omega\|_{m, \frac{1}{2}}=\left(\sum_{|\alpha| \leqslant m}\left\|D^{\alpha} u\right\|_{L^{2}(\Omega)}^{2}+|u|_{\Omega}^{2}\right)^{\frac{1}{2}}
$$


Si prova che esiste una costante $c>0$ tale che:

$$
\|u, \Omega\|_{m, \frac{1}{2}} \leqslant c \mid\|u, \Omega\|_{m, \frac{1}{2}}\left({ }^{2}\right) .
$$

Infine, detto $H^{m, 0}(\Omega)$ il completamento di $O_{x, t}^{m, 0}(\bar{\Omega})$ rispetto alla norma:

$$
\|u, \Omega\|_{m, 0}=\left(\sum_{|\alpha| \leqslant m}\left\|D^{\alpha} u\right\|_{L^{2}(\Omega)}^{2}\right)^{\frac{1}{3}},
$$

se $0 \leqslant \mu \leqslant 2 m+n$, denotiamo con $H^{m, 0, \mu}(\Omega)$ il sottospazio di $H^{m, 0}(\Omega)$ costituito dalle funzioni $u$ per le quali risulta:

$$
[u, \Omega]_{m, 0}^{(\mu)}=\sup _{\substack{(x, t) \in \bar{\Omega} \\ 0<Q<+\infty}}\left(Q^{-\mu} \iint_{I\left(x_{0}, t_{0} ; Q, 2,2 m\right)} \sum_{|\alpha|=m}\left|D^{\alpha} u\right|^{2} d x d t\right)^{k}<+\infty
$$

ove:

$$
I\left(x_{0}, t_{0} ; \varrho, 2 m\right)=\left\{(x, t) \in \bar{\Omega}:\left|x-x_{0}\right|<\varrho,\left|t-t_{0}\right|<\varrho^{2 m}\right\}
$$

\section{2. - Lemmi relativi a potenziali.}

In questo numero esponiamo alcuni lemmi concernenti potenziali relativi ad equazioni paraboliche che utilizzeremo, in seguito, per costruire una funzione verificante assegnate condizioni al contorno.

LemaA 2.1. - Sia $\left.\Omega=\boldsymbol{R}^{n} \times\right] 0, T[(T>0)$ e sia data l'equazione:

$$
\sum_{|\alpha| \leqslant a m} a_{\alpha}(x, t) D^{\alpha} u+\frac{\partial u}{\partial t}=0
$$

verificante le ipotesi seguenti:

I) $a_{\alpha} \in C_{x, i}^{\lambda, 0}(\bar{\Omega}) \quad(0<\lambda<1,|\alpha| \leqslant 2 m)$.

II) Le funzioni $a_{\alpha}(|\alpha|=2 m)$ sono continue rispetto a $t$, uniformemente al variare $d i(x, b) \in \bar{\Omega}$.

III) L'equazione (2.1) è uniformemente parabotica in $\bar{\Omega}$, cioè esiste un numero $\delta>0$ tale che:

$$
\left\{\begin{array}{l}
\operatorname{Re}\left\{(--1)^{m} \sum_{|\alpha|=2 m} a_{\alpha}(x, t) \xi^{\alpha}\right\}>\delta|\xi|^{2 m} \\
\forall(x, t) \in \bar{\Omega}, \forall \xi \in \boldsymbol{R}^{n}-\{0\}
\end{array}\right.
$$

(2) Cfr. [8], Prop. 3.9 e Def. 3.16. 
Detta $\Gamma(x, t ; \xi, \tau)$ la soluzione fondamentale dell'equazione $(2.1)$, se $\psi \in C^{m-1+\lambda}\left(\boldsymbol{R}^{\prime \prime}\right)$ e si pone:

$$
w(x, t)=\int_{\boldsymbol{R}^{n}} \Gamma(x, t ; \xi, 0) \psi(\xi) d \xi
$$

allora si ha:

$$
w \in C_{x, t}^{m-1+\lambda, \lambda / 2 m}(\bar{\Omega}) \cap C_{x, t}^{2 m, 1}(\Omega) \cap{ }_{R} W_{2}^{m, \frac{1}{2}}(A) \cap H^{m, 0, \mu}(A)
$$

ove $A=G \times] 0, T\left[, G\right.$ è un arbitrario insieme aperto limitato $d i \boldsymbol{R}^{n}, \mu=n+2 m+$ $+2 \lambda-2$.

Inoltre, risulta:

$$
|w, \bar{\Omega}|_{m-1,0}^{(\lambda, 2 m)}+\|w, A\|_{m, \frac{1}{2}} \frac{1}{5}[w, A]_{m, 0}^{(\mu)} \leqslant c\left|\psi, \boldsymbol{R}^{n}\right|_{m-1}^{(\lambda)}
$$

con e costante positiva dipendente dai coefficienti di (2.1), da $\lambda, T$ e da $A$.

Dmostraziont. - Da un nostro precedente risultato $\left({ }^{3}\right)$ consegue, nelle ipotesi fatte, che $w \in C_{\alpha, t}^{m-1+\lambda, \lambda / 2 m}(\bar{\Omega})$ e che:

$$
|w, \bar{\Omega}|_{m-1,0}^{(\lambda, 2 m)} \leqslant c\left|\psi, \boldsymbol{R}^{n}\right|_{m-1}^{(\lambda)}
$$

Utilizzando, ancora, nostri risultati si prova che:

$$
[w, A]_{m, 0}^{(\mu)} \leqslant c\left|\psi, \boldsymbol{R}^{n}\right|_{m-1}^{(2)}
$$

In effetti, si ha:

$$
\left|D^{\alpha} w(x, t)\right| \leqslant c\left|\psi, \boldsymbol{R}^{n}\right|_{m-1}^{(\lambda)} \cdot{ }^{(\lambda-1) / 2 m} \quad \forall(x, t) \in \Omega(|\alpha|=m)\left(^{4}\right)
$$

da cui consegue facilmente la (2.3).

Proviamo, infine, che:

$$
\|w, A\|_{m, \frac{1}{2}} \leqslant c\left|\psi, \boldsymbol{R}^{n}\right|_{m-1}^{(\lambda)}
$$

Prolunghiamo, innanzitutto, $w$ in $\boldsymbol{R}^{n+1}$, ponendo:

$$
w_{1}(x, t)= \begin{cases}w(x, T) & \forall t \geqslant T \\ w(x, t) & \forall t \in] 0, T[ \\ \psi(x) & \text { se } t=0 \\ w(x,-t) & \forall t<0\end{cases}
$$

(3) Cfr. [4], Prop. IX.

(4) Cfr. [4], Prop. VI. 
Sia $\chi \in \mathscr{D}(\boldsymbol{R})$ con $\chi(t)=1 \forall t \in[0, T]$ e sia $[a, b]$ un intervallo contenente il supporto di $\chi$.

Ponendo $P w=\chi w_{1}$ otteniamo, quindi, un particolare prolungamento continuo di $w\left({ }^{(5)}\right.$.

Orbene, risulta:

$$
\| P w, G \times]-\infty,+\infty\left[\|_{m, 3} \leqslant c\left|\psi, \boldsymbol{R}^{n}\right|_{m-1}^{(\hat{\lambda})} .\right.
$$

In effetti, per proprietà della trasformazione di FourIER $\left(^{6}\right)$, si ha:

$$
\begin{aligned}
\int_{\sigma} d x \int_{-\infty}^{+\infty}|t| \cdot|\mathcal{F}(P w)|^{2} d t=0 \int_{\sigma} d x \int_{-\infty}^{+\infty}\left|\mathcal{F}\left(\frac{d}{d t} \int_{a}^{t} P w(x, \sigma)(t-\sigma)^{-\frac{1}{2}} d \sigma\right)\right|^{2} d t= \\
=c \int_{\sigma} d x \int_{-\infty}^{+\infty} \mid \frac{d}{d t} \int_{a}^{t} P w(x, \sigma)(t-\sigma)^{-\frac{1}{2}} d \sigma^{2} d t
\end{aligned}
$$

D'altra parte, tenendo presente che:

$$
\left\{\begin{array}{l}
|w(x, t)| \leqslant c\left|\psi, \boldsymbol{R}^{n}\right|_{m-1}^{(\lambda)} \\
\left|\frac{\partial w}{\partial t}(x, t)\right| \leqslant c \mid \psi, \boldsymbol{R}_{\mid m-1}^{n(\lambda)} \cdot t^{(\lambda-2 m) / 2 m}
\end{array} \quad \forall(x, t) \in \Omega\left(^{7}\right)\right.
$$

(5) Si tenga presente che, nelle ipotesi fatte, ̀̀ $\lim _{t \rightarrow 0^{+}} w(x, t)=\psi(x)$ (cfr. [6]).

( $\left.{ }^{(}\right)$Per $f \in L^{2}(\boldsymbol{R}), f=0$ per $t<a$, introdotta la derivata frazionaria di $f$, ordine $\varrho$ :

$$
D^{\varrho} f(x, t)=\frac{1}{\Gamma(1-\varrho)} \frac{d}{d t} \int_{a}^{t} f(\eta)(t-\eta)^{-\varrho} d \eta \quad(0<\varrho<1)
$$

si prova, utilizzando la teoria delle distribuzioni, che:

$$
\mathscr{F}\left(D^{\varrho} f\right)=\exp \left[i \varrho \frac{\pi}{2}\right] \exp \left[i_{\varrho} \theta\right]|\eta| e \widetilde{F}(f) \quad(\theta=0 \text { se } \eta>0, \theta=\pi \text { se } \eta<0) .
$$

(La funzione $x^{-0}$, che compare nell'integrale, si intende prolungata per $x \leqslant 0$, ponendola eguale a zero), Cfr. [7], [10].

(7) Cfr. [4], Prop. VI, tenendo anche presente che:

$$
\frac{\partial w}{\partial t}=-\sum_{|\alpha| \leqslant 2 m} a_{\alpha} D^{\alpha} w \quad \text { in } \Omega .
$$


si ottiene:

$$
\begin{aligned}
& \left|\frac{d}{d t} \int_{a}^{t} P w(x, \sigma)(t-\sigma)^{-\frac{1}{2}} d \sigma\right|=2\left|\frac{d}{d t} \int_{a}^{t}(t-\sigma)^{\frac{1}{2}} \frac{\partial P w}{\partial \sigma} d \sigma\right|= \\
& =\left|\int_{a}^{t}(t-\sigma)^{-\frac{1}{2}}\left(\chi^{\prime} \cdot w_{1}+\chi \frac{\partial w_{1}}{\partial \sigma}\right) d \sigma\right| \leqslant \\
& \leqslant o\left|\psi, \boldsymbol{R}^{n}\right|_{m-1}^{(\lambda)}+o\left|\psi, \boldsymbol{R}^{n}\right|_{m-1}^{(\lambda)} \cdot \int_{a}^{t}(t-\sigma)^{-\frac{1}{2}|\sigma|^{(\lambda-2 m) 2 m}} d \sigma \leqslant \\
& \leqslant o\left|\psi, \boldsymbol{R}^{n}\right|_{m-1}^{(\lambda)}\left(1+|t|^{(\lambda-m) / 2 m}\right) \quad(\forall t \neq 0)\left({ }^{8}\right)
\end{aligned}
$$

mentre:

$$
\begin{aligned}
\left|\frac{d}{d t} \int_{a}^{t} P w(x, \sigma)(t-\sigma)^{-\frac{1}{2}} d \sigma\right| & =\frac{1}{2}\left|\int_{a}^{b} P w(x, \sigma)(t-\sigma)^{-\frac{3}{2}} d \sigma\right| \leqslant \\
& \leqslant c\left|\psi, \boldsymbol{R}^{n}\right|_{m-1}^{(\lambda)}(t-b)^{-\frac{3}{2}} \quad(\forall t>b) .
\end{aligned}
$$

Da (2.7), (2.8), (2.9) si deduce immediatamente:

$$
\|P w\|_{0, \frac{1}{2}}=\frac{1}{2 \pi}\left(\int_{G} d x \int_{-\infty}^{+\infty}|t||\mathscr{F}(P w)|^{2} d t\right)^{\frac{1}{2}} \leqslant c\left|\psi, \boldsymbol{R}^{n}\right|_{m-1}^{(\lambda)} .
$$

Da questo risultato e dalla (2.4) consegue la (2.6) e da questa, tenendo presenti le definizioni del n. 1, infine, la (2.5).

Da (2.2), (2.3), (2.5), essendo noto che $w \in O_{x, t}^{2 m, 1}(\Omega)$, si deduce la tesi.

Lemma 2.2. - Posto $\boldsymbol{R}_{+}^{n}=\left\{x \in \boldsymbol{R}^{n}: x_{n}>0\right\}$, siano $\mathfrak{G}_{j}(x, t)\left(j=1, \ldots, m ; x \in \boldsymbol{R}_{+}^{n}, t \in \boldsymbol{R}\right)$ $i$ nuclei di Poisson relativi al problema di Dirichlet nel semispazio $\boldsymbol{R}_{+}^{n} \times \boldsymbol{R}$ per un'arbitraria equazione parabolica di ordine $2 m$, a coefficienti costanti, del tipo $(2.1)\left({ }^{9}\right)$.

${ }^{(8)}$ Ponendo $\sigma=t \eta$, si trova, infatti, facilmente:

$$
\begin{array}{ll}
\int_{a}^{t}(t-\sigma)^{-\frac{1}{2}}|\sigma|^{\lambda / 2 m-1} d \sigma \leqslant t^{(\lambda-m) / 2 m} \int_{-\infty}^{1}(1-\eta)^{-\frac{1}{2}}|\eta|^{\lambda / 2 m-1} d \eta & \text { se } t>0, \\
\int_{a}^{t}(t-\sigma)^{-\frac{1}{2}}|\sigma|^{\lambda / 2 m-1} d \sigma \leqslant|t|^{(\lambda-m) / 2 m} \int_{1}^{+\infty}(\eta-1)^{-\frac{1}{2}}|\eta|^{\lambda / 2 m-1} d \eta & \text { se } t<0 .
\end{array}
$$

(9) Ci riferiamo ai nuclei introdotti in [11], formula (2.69). 
Siano, inoltre, assegnate $m$ funzioni:

$$
\varphi_{j} \in C_{x^{\prime}, t}^{m-j+\lambda_{2} \lambda_{12 m}}\left(\boldsymbol{R}^{n-1} \times[0, T]\right) \quad(j=1, \ldots, m ; 0<\lambda<1, T>0)
$$

verificanti la condiaione:

$$
\varphi_{j}\left(x^{\prime}, 0\right)=0 \quad\left(j=1, \ldots, m ; x^{\prime} \in \boldsymbol{R}^{n-1}\right) .
$$

Posto:

$$
\begin{gathered}
v=\sum_{j=1}^{m} v_{j} \\
v_{j}(x, t)=\int_{\boldsymbol{R}^{n-1}} d y^{\prime} \int_{0}^{t} \mathcal{G}_{j}\left(x^{\prime}-y^{\prime}, x_{n}, t-\theta\right) \varphi_{j}\left(y^{\prime}, \theta\right) d \theta
\end{gathered}
$$

risulta:

$$
v \in C_{x, t}^{m-1+\lambda, \lambda / 2 m}(\bar{A}) \cap C_{x, t}^{2 m, 1}(A) \cap_{g} W_{2}^{m, \frac{1}{2}}(A) \cap H^{m, 0, \mu}(A)
$$

ove $A=G \times] 0, T\left[, G\right.$ è un arbitrario insieme aperto limitato di $\boldsymbol{R}_{+}^{n}, \mu=n+2 m+$ $+2 \lambda-2$.

Inoltre, risulta:

$$
\begin{array}{ll}
\frac{\partial^{j-1} v}{\partial x_{n}^{j-1}}\left(x^{\prime}, 0, t\right)=\varphi_{j}\left(x^{\prime}, t\right) & \left(j=1, \ldots, m ;\left(x^{\prime}, t\right) \in \boldsymbol{R}^{n-1} \times[0, T]\right) \\
v\left(x^{\prime}, x_{n}, 0\right)=0 & \left(\left(x^{\prime}, x_{n}\right) \in \boldsymbol{R}_{+}^{n}\right) \\
\left|D^{\alpha} v(x, t)\right| \leqslant c t^{(m-1-|\alpha|+\lambda) / 2 m} \Phi_{m-1,0}^{(\lambda)} & \left(|\alpha| \leqslant m-1 ;(x, t) \in \boldsymbol{R}_{+}^{n} \times[0, T]\right) \\
\left|D^{\alpha} v(x, t)\right| \leqslant c x_{n}^{m-1-|\alpha|+\lambda} \Phi_{m-1,0}^{(\lambda)} & \left(|\alpha| \geqslant m ;(x, t) \in \boldsymbol{R}_{+}^{n} \times[0, T]\right) \\
|v, \bar{A}|_{m-1,0}^{(\lambda, 2 m)}+\|v, A\|_{m, \frac{1}{2}}+[v, A]_{m, 0}^{(\mu)} \leqslant c \Phi_{m-1,0}^{(\lambda)} &
\end{array}
$$

dove:

$$
\Phi_{m-1,0}^{(\lambda)}=\sum_{j=1}^{m}\left|\varphi_{j}, \boldsymbol{R}^{n-1} \times[0, T]\right|_{m-j, 0}^{(\lambda, 2,2 m)}
$$

con c costante positiva dipendente, oltre che dai coefficienti dell'equazione, da $\lambda, T$ e $d a \mathrm{~A}$.

Dinostrazione. - La dimostrazione di questo lemma si consegue facilmente, integrando nostri precedenti risultati $\left({ }^{10}\right)$ con considerazioni analoghe a quelle adoperate per il Lemma 2.1.

(10) Cfr. [3], Lemma 2.1. 
Tuttavia, per comodità del lettore, ne daremo qualche cenno. Innanzitutto, osserviamo che le $\varphi_{j}(j=1, \ldots, m)$ si possono prolungare per $t<0$ (ponendole eguali a zero) e per $t>T$, in modo da conservare le loro proprietà di regolarità.

Per i risultati del citato lavoro di O. A. SolonNLKov [11] è, allora, verificata la (2.12); la (2.13) è ovvia. Allo scopo di provare (2.14), (2.15) ricordiamo alcune proprietà dei nuclei $\mathscr{G}_{j}(x, t)$ dimostrate da SolonNrKov in [11].

Le $\mathfrak{G}_{j}(x, t)(j=1, \ldots, m)$ sono infinitamente differenziabili, per $x_{n}>0$, sono nulle per $t<0$ e verificano le disuguaglianze:

$$
\left\{\begin{array}{l}
\mid D^{\alpha} \frac{\partial^{r}}{\partial t^{r}} \mathcal{S}_{j}(x, t) \leqslant 0 t^{-\frac{\chi_{j}^{\alpha}, r}{2 m}} \exp \left(-\omega \frac{|x|^{2 m /(2 m-1)}}{t^{1 /(2 m-1)}}\right) \\
\text { ove: } \\
\chi_{j}^{\alpha_{i} t}=n+2 m-j+|\alpha|+2 m r, \quad \omega>0 .
\end{array}\right.
$$

Inoltre, può seriversi:

$$
\mathcal{G}_{j}(x, t)=\Lambda_{x^{\prime}, t}^{(k)} \mathcal{G}_{j}^{(k)}\left(x^{\prime}, x_{n}, t\right)
$$

ove $A_{x^{\prime}, t}^{(k)}$ è I'iterato $k$-esimo dell'operatore:

$$
A_{x^{2} . t}=\frac{\partial}{\partial t}+(-1)^{m}\left(1+\delta_{1}\right) A_{x^{\prime}}^{(m)} \quad\left(\delta_{1}>0\right)
$$

avendosi, inoltre:

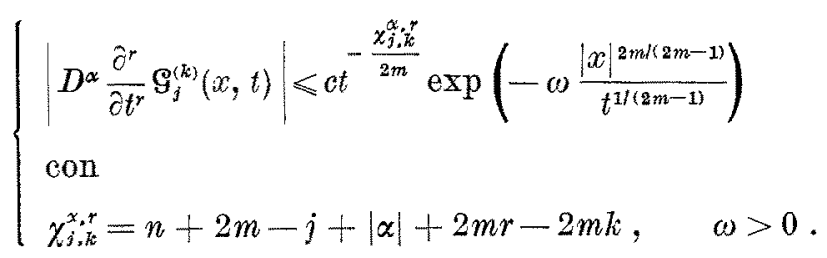

Ciò premesso, tenendo presente la $(2.16)$, per $x_{n}>0$ e per ogni $\alpha$ si ba:

$$
\begin{aligned}
D^{\alpha} v_{j}(x, t)=\int_{\mathbf{R}^{n-1}} d y^{\prime} \int_{0}^{t} D^{\alpha} \mathcal{G}_{j}\left(x^{\prime}\right. & \left.-y^{\prime}, x_{n}, t-\theta\right)\left[\varphi_{j}\left(y^{\prime}, \theta\right)-\varphi_{j}\left(y^{\prime}, t\right)\right] d \theta+ \\
& +\int_{\mathbf{R}^{n-1}} D^{\alpha} \mathcal{G}_{j}^{(1)}\left(x^{\prime}-y^{\prime}, x_{n}, t\right) \varphi_{j}\left(y^{\prime}, t\right) d y^{\prime}+ \\
& +(-1)^{m}\left(1+\delta_{1}\right) \int_{\mathbf{R}^{n-1}} d y^{\prime} \int_{0}^{t} \Delta_{x^{\prime}}^{(m)} D^{\alpha} \mathcal{G}_{j}^{(\mathbf{1})}\left(x^{\prime}-y^{\prime}, x_{n}, t-\theta\right) \varphi_{j}\left(y^{\prime}, t\right) d \theta= \\
& =I_{1}^{(j)}+I_{2}^{(j)}+I_{3}^{(j)} .
\end{aligned}
$$


Maggioriamo l'integrale $I_{1}^{(j)}$. Da (2.10), (2.17) si deduce:

$$
\left|I_{1}^{(j)}\right| \leqslant c \Phi_{m-1,0}^{(\lambda)} \int_{\boldsymbol{R}^{n-1}} d y^{\prime} \int_{0}^{t}(t-\theta)^{-(n+m+|\alpha|-\lambda) / 2 m} \exp \left\{-\omega \frac{\left(\left|x^{\prime}-y^{\prime}\right|^{2}+x_{n}^{2}\right)^{m /(2 m-1)}}{(t-\theta)^{1 / 2 m-1)}}\right\} d \theta
$$

Da questa disuguaglianza, tenendo presenti alcuni nostri risultati $\left({ }^{11}\right)$, consegue:

$$
\left|I_{1}^{(j)}\right| \leqslant c t^{(m-1-[\alpha \mid+\lambda) / 2 m} \Phi_{m-1,0}^{(j)} \quad \text { se }|\alpha| \leqslant m-1 .
$$

Se $|\alpha| \geqslant m$, effettuando in $(2.20)$ il cambiamento di variabili:

$$
t-\theta=\eta x_{n}^{2 m}, \quad x_{i}-y_{i}=x_{n} \eta^{1 / 2 m} \xi_{i} \quad(i=1, \ldots, n-1)
$$

si ha:

$$
\begin{aligned}
\left|I_{\mathbf{1}}^{(j)}\right| \leqslant c x_{n}^{m-1-|\alpha|+\lambda} \Phi_{m-1,0}^{(\lambda)} \int_{\boldsymbol{R}^{n-1}} d \xi^{\prime} \int_{0}^{t / x_{n}^{z_{m}}} \eta^{-(m+1+|\alpha|-\lambda) / 2 m} \exp \left\{-\omega\left(\left|\xi^{\prime}\right|^{2}+\frac{1}{\eta^{1 / m}}\right)^{m /(2 m-1)}\right\} d \eta \leqslant \\
\leqslant e x_{n}^{m-1-|\alpha|+\lambda} \Phi_{m-1,0}^{(2)}
\end{aligned}
$$

Maggioriamo l'integrale $I_{\mathrm{a}}^{(j)}$. Da $(2.10),(2.11),(2.19)$ consegue:

$$
\left|I_{2}^{(j)}\right| \leqslant e \Phi_{m-1,0}^{(\lambda)} t^{(m-n-|\alpha|+\lambda) / 2 m} \int_{\mathbf{R}^{n-1}} \exp \left\{-\omega \frac{\left(\left|x^{\prime}-y^{\prime}\right|^{2}+x_{n}^{2}\right)^{m /(2 m-1)}}{t^{1 /(2 m-1)}}\right\} d y^{\prime}
$$

da cui, col cambiamento di variabili

$$
x_{i}-y_{i}=\xi_{i} t^{1 / 2 m} \quad(i=1, \ldots, n-1)
$$

si ottiene:

$$
\begin{aligned}
&\left|I_{2}^{(j)}\right| \leqslant c \Phi_{m-1,0}^{(\lambda)} t^{(m-1-|\alpha|+\lambda) / 2 m} \int_{\boldsymbol{R}^{n-1}} \exp \left\{-\omega\left(\left|\xi^{\prime}\right|^{2}+\frac{\hat{x}_{n}^{2}}{t^{1 / m}}\right)^{m /(2 m-1)}\right\} d \xi^{\prime} \leqslant \\
& \leqslant \omega \Phi_{m-1,0}^{(\lambda)} t^{(m-1-|\alpha|+\lambda) / 2 m} \cdot \exp \left(-\frac{\omega}{2} \frac{x_{n}^{2 m /(2 m-1)}}{t^{1 /(2 m-1)}}\right)
\end{aligned}
$$

e, quindi, immediatamente:

$$
\begin{array}{ll}
\left|I_{2}^{(j)}\right| \leqslant c t^{(m-1-|\alpha|+\lambda) / 2 m} \Phi_{m-1,0}^{(\lambda)} & \text { se }|\alpha| \leqslant m-1 \\
\left|I_{2}^{(j)}\right| \leqslant c x_{n}^{m-1-|\alpha|+\lambda} \Phi_{m-1,0}^{(\hat{j})} & \text { se }|\alpha| \geqslant m .
\end{array}
$$

(11) Cfr. [5], Prop. I. 
Maggioriamo l'integrale $I_{3}^{(j)}$. Integrando per parti e tenendo presente che:

$$
\int_{\boldsymbol{R}^{n-1}} d y^{\prime} \int_{0}^{t} D_{v^{\beta}}^{\beta} D^{x} \mathcal{G}_{j}^{(1)}\left(x^{\prime}-y^{\prime}, x_{n}, t-\theta\right) d \theta=0 \quad\left(x_{n}>0\right)
$$

ove $D_{y^{\prime}}^{\beta}$ è una derivata di ordine $|\beta|>0$ rispetto alle variabili $y_{1}, \ldots, y_{n-1}$, si ottiene:

$I_{3}^{(j)}=\sum_{i=1}^{N} \int_{\mathbf{R}^{n-1}} d y^{\prime} \int_{0}^{t} R_{i}^{(1)}\left(\frac{\partial}{\partial x^{\prime}}\right) D^{\alpha \mathcal{G}_{j}^{(1)}}\left(x^{\prime}-y^{\prime}, x_{n}, t-\theta\right)\left[R_{i}^{(2)}\left(\frac{\partial}{\partial y^{\prime}}\right) \varphi_{j}\left(y^{\prime}, t\right)-R_{i}^{(2)}\left(\frac{\partial}{\partial x^{\prime}}\right) \varphi_{j}\left(x^{\prime}, t\right)\right] d \theta$ ove $N$ è il numero dei termini del polinomi $\left(\sum_{i=1}^{n-1} x_{i}^{2}\right)^{m}, R_{i}^{(1)}$ è un operatore differenziale di ordine $m+j, R_{i}^{(2)}$ è un operatore differenziale di ordine $m-j$.

Da (2.10), (2.19) si ha:

$$
\left|I_{\mathbf{a}}^{(j)}\right| \leqslant c \Phi_{m-1,0}^{(\lambda)} \int_{\boldsymbol{R}^{n-1}} d y^{\prime} \int_{0}^{t}\left|x^{\prime}-y^{\prime}\right|^{\lambda}(t-\theta)^{-(m+m+m+|\alpha|) / 2 m} \exp \left\{-\omega \frac{\left(\left|x^{\prime}-y^{\prime}\right|^{2}+x_{n}^{2}\right)^{m /(2 m-1)}}{(t-\theta)^{1 / 2 m-1)}}\right\} d \theta
$$

Procedendo come per $I_{1}^{(j)}$, si ha:

$$
\begin{array}{ll}
\left|I_{3}^{(j)}\right| \leqslant c t^{(m-1-|\alpha|+\lambda) / 2 m} \cdot \Phi_{m-1,0}^{(\lambda)} & \text { se }|\alpha| \leqslant m-1 \\
\left|I_{3}^{(j)}\right| \leqslant c x_{n}^{m-1-|\alpha|+\lambda} \cdot \Phi_{m-1,0}^{(\lambda)} & \text { se }|\alpha| \geqslant m .
\end{array}
$$

Dalle disuguaglianze $(2.21), \ldots,(2.26)$ conseguono le formule $(2.14),(2.15)$ che intendevamo dimostrare.

Per provare, infine, la (2.16), osserviamo che essendo dimostrata, nel citato lavoro di SoLonvirov, la disuguaglianza:

$$
\mid v, \bar{A}_{m-1,0}^{(\lambda, 2 m)} \leqslant c \Phi_{m-1,0}^{(\lambda)}
$$

è sufficiente provare che:

$$
\begin{aligned}
& \|v, A\|_{m, \frac{1}{2}} \leqslant c \Phi_{m-1,0}^{(\alpha)} \\
& {[v, A]_{m, 0}^{(\mu)} \leqslant c \Phi_{m-1,0}^{(\lambda)} .}
\end{aligned}
$$

Per dimostrare la (2.27), denotiamo con $P v$ il prolungamento di $v$ ottenuto ponendo:

$$
P v(x, t)=\left\{\begin{array}{ll}
0 & \forall t<0 \\
v(x, t) & \forall t \in[0, T] \\
v(x, 2 T-t) & \forall t>T
\end{array} \quad\left(\forall x \in \mathbf{R}_{+}^{n}\right)\right.
$$


Per il teorema della media e per la (2.14), posto $\varrho=(m-1+\lambda) / 2 m$ si ha:

$$
\begin{aligned}
\left|\frac{d}{d t} \int_{0}^{t} P v(x, \sigma)(t-\sigma)^{-\frac{1}{2}} d \sigma\right| \leqslant c \Phi_{m-1,0}^{(\lambda)}\left|\frac{d}{d t} \int_{0}^{t} \sigma^{e}(t-\sigma)^{-\frac{1}{d}} d \sigma\right| \leqslant & \\
\leqslant c \Phi_{m-1,0}^{(\lambda)}\left|\frac{d}{d t} t^{\frac{1}{1}+e} \int_{0}^{t} \eta^{e}(1-\eta)^{-\frac{1}{d}} d \eta\right| \leqslant e \Phi_{m-1,0}^{(\lambda)} t^{-\frac{1}{2}+e} & (\forall t>0) \\
\left|\frac{d}{d t} \int_{0}^{t} P v(x, \sigma)(t-\sigma)^{-\frac{1}{2}} d \sigma\right| \leqslant c \Phi_{m-1.0}^{(\lambda)}(t-2 T)^{-\frac{3}{2}} & (\forall t>2 T) .
\end{aligned}
$$

In base a $(2.29)$ e (2.30), ragionando come nella dimostrazione della (2.5) del lemma precedente, si prova la (2.27).

Dalla (2.15) consegue, facilmente, la (2.28).

Le proprietà qualitative di $v$ sono, infine, conseguenza di quanto dimostrato innanzi oppure di proprietà dimostrate nel citato lavoro di SoLONNTKov [11].

\section{3. - Posizione del problema e relativi teoremi di esistenza ed unicità.}

Sia $G$ un aperto limitato di $\boldsymbol{R}^{n}, T$ un numero reale positivo. Poniamo

$$
\Omega=G \times] 0, T\left[, \quad \Omega^{\prime}=\partial G \times\right] 0, T[
$$

dove $\partial G$ è la frontiera di $G$, che supporremo infinitamente differenziabile.

Consideriamo la forma bilineare:

$$
B(u, \tau)=\int_{0}^{T} d t \int_{a}\left[\sum_{|p|,|q| \leqslant m}(-1)^{|p|} a_{p q}(x, t) D^{q} u D^{p} \bar{\tau}-u \frac{\partial \bar{\tau}}{\partial t}\right] d x
$$

nell'ipotesi che sia:

$$
a_{p, q} \in C_{x, t}^{|p|+\lambda, 0}(\bar{\Omega}) \quad(|p|,|q| \leqslant m) .
$$

Supponiamo, anche, che la forma (3.1) sia parabolica, cioè che esista un numero reale $\delta>0$ tale che:

$$
\left\{\begin{array}{c}
\operatorname{Re}\left\{\left(-1^{m} \sum_{|p| .|a|=m} a_{x a}(x, t) \xi^{p+a}\right\}>\delta|\xi|^{2 m}\right. \\
\forall \xi \in \boldsymbol{R}^{n}-\{0\}, \forall(x, t) \in \bar{\Omega}
\end{array}\right.
$$


Consideriamo, inoltre, la forma bilineare:

$$
F(f, \tau)=\sum_{|\alpha| \leqslant m} \int_{0}^{T} d t \int_{\sigma} f_{\alpha} \cdot D^{\alpha} \tilde{\tau} d x
$$

ove $f=\left(f_{\alpha}\right)_{|x| \leqslant m}$ è un assegnato sistema di funzioni verificanti la condizione seguente:

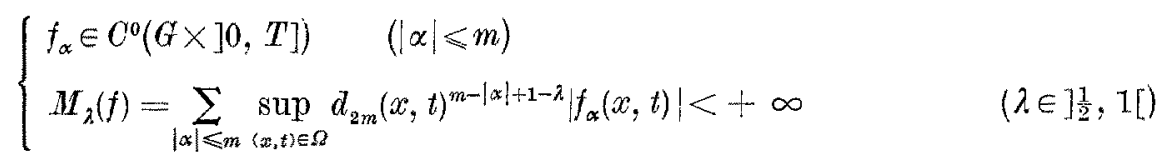

dove:

$$
d_{2 m}(x, t)=\inf \left\{d(x), t^{1 / 2 m}\right\} \quad \forall(x, t) \in \Omega
$$

avendo denotato con $d(x)$ la distanza di $x \in G$ da $\partial G$.

Siano assegnate anche $m$ funzioni:

$$
\varphi_{j} \in O_{x^{\prime}, t}^{m-j+\lambda, \lambda / 2 m}\left(\bar{\Omega}^{\prime}\right) \quad(j=1, \ldots, m)\left({ }^{12}\right)
$$

ed una funzione:

$$
\psi \in O^{m-1+\lambda}(\bar{Q})
$$

in modo che sia soddisfatta la condizione di compatibilità:

$$
\frac{d^{j-1} \psi}{d \nu^{j-1}}\left(x^{\prime}\right)=\varphi_{j}\left(x^{\prime}, 0\right) \quad\left(j=1, \ldots, m ; x^{\prime} \in \hat{\imath} G\right)
$$

essendo $\nu$ la normale interna nel punto $x^{\prime} \in \partial G$. Lo scopo principale di questo lavoro è lo studio del problema:

$$
\begin{cases}B(u, \tau)=F(f, \tau) & \forall \tau \in \mathcal{D}(\Omega) \\ \frac{d^{j-1} u}{d p^{j-1}}\left(x^{\prime}, t\right)=\varphi_{j}\left(x^{\prime}, t\right) & \left(j=1, \ldots, m ;\left(x^{\prime}, t\right) \in \Omega^{\prime}\right) \\ u(x, 0)=\psi(x) & (x \in G)\end{cases}
$$

nelle ipotesi innanzi precisate.

È essenziale, a tale proposito, il lemma seguente.

(12) Le definizioni di $C_{x^{\prime}, t}^{m-j+\lambda, \lambda / 2 m}\left(\Omega^{\prime}\right)$ e della relativa norma $\left|\cdot, \bar{\Omega}^{\prime}\right|_{m-i, 0}^{(\lambda, 2 m)}$ si riconducono, nel modo consueto, alle analoghe definizioni relative al caso in cui linsieme di definizione $\partial$ del tipo $A \times[0, T]$ con $A$ aperto di $\boldsymbol{R}^{n-1}$. 
LeMma 3.1. - Se $f=\left(f_{\alpha}\right)_{|\alpha| \leqslant m}$ è un sistema dì funzioni verificante l'ipotesi (3.4) allora $\tau \rightarrow F(f, \tau)$ è un funzionale lineare e continuo in ${ }_{R}^{0} W_{2}^{m, \frac{1}{2}}(\Omega)$.

Inoltre se, per $\varepsilon$ reale positivo ed abbastanza piccolo, si pone:

$$
\Omega_{\varepsilon}=\left\{(x, t) \in \Omega: d_{2 m}(x, t) \leqslant \varepsilon\right\}
$$

risulta, $\forall \tau \in \in_{R}^{0} W_{2}^{m, \frac{1}{2}}(\Omega)$ :

$$
\left.|F(f, \tau)| \leqslant c\left\{\sum_{|\alpha| \leqslant m} \sup _{(x, t) \in \Omega, \Omega-\Omega_{\varepsilon}}\left|f_{\alpha}(x, t)\right|+\varepsilon^{\lambda-\frac{1}{2}} M_{\lambda}(f)\right\}\right\} \| \tau,\left.\Omega\right|_{m, \frac{1}{3}}
$$

con o costante positiva dipendente da $m, \lambda, \Omega$.

Dmostraziont. - Ovviamente, è sufficiente provare la (3.9). Posto:

$$
\begin{aligned}
& \Omega_{\varepsilon_{,} t}=\left\{(x, t) \in \Omega_{\varepsilon}: d_{2 m}(x, t)=t^{1 / 2 m}\right\} \\
& \Omega_{\varepsilon_{x, x}}=\left\{(x, t) \in \Omega_{\varepsilon}: d_{2 m}(x, t)=\vec{a}(x)\right\}
\end{aligned}
$$

poichè:

$$
\iint_{\Omega} f_{\alpha} D^{\alpha} \tilde{\tau} d x d t=\iint_{\Omega-\Omega_{\varepsilon}} f_{\alpha} D^{\alpha} \bar{\tau} d x d t+\iint_{\Omega_{\varepsilon}} f_{\alpha} D^{\alpha} \bar{\tau} d x d t
$$

e, d'altra parte, ovviamente, si ha:

$$
\left|\iint_{\Omega} f_{\alpha} D^{\alpha} \bar{\tau} d x d t\right| \leqslant 0 \sup _{(\alpha, t) \in \Omega-\Omega_{\varepsilon}}\left|f_{\alpha}(x, t)\right| \cdot \mid\left[D^{\alpha} \tau \|_{L^{*}(\Omega)} \quad(|\alpha| \leqslant m)\right.
$$

sarà sufficiente provare che, per $|\alpha| \leqslant m, \forall \tau \in \mathcal{D}(\Omega)$, risulta:

$$
\left\{\begin{array}{l}
\left|\iint_{\Omega_{\varepsilon, x}} f_{\alpha} \cdot D^{\alpha} \bar{\tau} d x d t\right| \leqslant c \varepsilon^{\lambda-\frac{1}{2}} M_{\lambda}(f) \cdot \sum_{|\alpha| \leqslant m}\left\|D^{\alpha} \tau\right\|_{L^{2}(\Omega)} \\
\left|\iint_{\Omega_{\varepsilon, t}} f_{\alpha} \cdot D^{\alpha} \bar{\tau} d x d t\right| \leqslant e \varepsilon^{\lambda-\frac{1}{2}} M_{\lambda}(f) \cdot\|\tau, \Omega\|_{m, \frac{1}{2}} .
\end{array}\right.
$$

Per dimostrare la prima delle (3.10) si può seguire, con qualche variante, il procedimento adoperato da C. Mrrarda in un caso analogo $\left({ }^{13}\right)$.

Dimostriamo, dunque, la seconda delle (3.10).

(13) Cfr. [9], dimostrazione della (10.2). 
Se $|\alpha|>0, \forall \tau \in \mathscr{D}(\Omega)$ si ha, facilmente:

$$
\begin{aligned}
\left|\iint_{\Omega_{\varepsilon, t}} f_{\alpha} D^{\alpha} \bar{\tau} d x d t\right| \leqslant c M_{\lambda}(f) \iint_{\Omega_{\varepsilon, t}} d_{2 m}(x, t)^{|\alpha|-m+\lambda-1}\left|D^{\alpha} \tau\right| d x d t \leqslant \\
\\
\quad \leqslant c M_{\lambda}(f)\left\|D^{\alpha} \tau\right\|_{L^{2}(\Omega)}\left(\int_{0}^{\varepsilon^{2 m}} t^{(|\alpha|-m+\lambda-1) / m} d t\right)^{\frac{1}{2}} \leqslant c M_{\lambda}(f) \varepsilon^{\lambda-\xi}\left\|D^{\alpha} \tau\right\|_{L^{2}(\Omega)}
\end{aligned}
$$

da cui consegue la seconda delle (3.10) (per $|\alpha|>0$ ).

Se $|\alpha|=0$, cominciamo col porre:

$$
\begin{aligned}
& t_{2 m a}(x)=\inf \left(d(x)^{2 m}, \varepsilon^{2 m}\right) \\
& \forall x \in G\left({ }^{14}\right) \\
& f^{*}(x, t)=\left\{\begin{array}{lll}
f_{\alpha}(x, t) & \text { se } 0<t<t_{2 m}(x) & \forall x \in G \\
0 & \text { se } t \leqslant 0, t \geqslant t_{2 m}(x) & \forall x \in G\left({ }^{15}\right)
\end{array}\right. \\
& \varrho=\frac{2 m-1}{4 m} \\
& h(x, t)=I^{\left(\frac{1}{2}-\varrho\right)}\left(f^{*}\right)=\frac{1}{\Gamma\left(\frac{1}{2}-\varrho\right)} \int_{0}^{t} f^{*}(x, \eta)(t-\eta)^{-\frac{1}{2}-\varrho} d \eta \quad((x, t) \in G \times \boldsymbol{R})\left({ }^{16}\right) .
\end{aligned}
$$

Proviamo, innanzitutto, che risulta:

$$
|h(x, t)| \leqslant \begin{cases}c M_{\lambda}(f) t^{-o+(\lambda-1) / 2 m} & \text { se } 0<t<t_{2 m}(x) \\ c M_{\lambda}(f) t_{2 m}(x)^{\beta} t^{-0-\beta+(\lambda-1) / 2 m} & \text { se } t \geqslant t_{2 m}(x)\end{cases}
$$

comunque si scelga il numero $\beta$ tale che:

$$
0<\frac{m+\lambda-1}{2 m}-\varrho<\beta<\frac{m+\lambda-1}{2 m} .
$$

(14) Osserviamo che:

$$
\left((x, t) \in Q_{\varepsilon, t}\right) \Leftrightarrow\left(x \in G, 0<t<t_{2 m}(x)\right) .
$$

${ }^{\left({ }^{5}\right)}$ Osserviamo che $t^{*}$ è sommabile (come funzione di $t$ ) in ogni intorno di $t=0$, dato che risulta:

$$
\left|f^{*}(x, t)\right| \leqslant c M_{\lambda}(f) \cdot t^{(-m+\lambda-1) / 2 m}
$$

(16) $I^{\left(\frac{1}{2}-Q\right)}\left(f^{*}\right)$ denota la primitiva di ordine frazionario $\frac{1}{2}-\varrho$ di $f^{*}$ (la funzione $x^{-\frac{1}{2}-Q}$, che compare nell'integrale, si intende prolungata per $x \leqslant 0$ ponendola eguale a zero). Cfr. ad esempio, [7], vol. I, p. 113. 
In effetti, se $0<t<t_{2 m}(x)$, da (3.4) si ha:

$$
\begin{aligned}
&|h(x, t)| \leqslant c \lambda I_{\lambda}(f) \int_{0}^{t} d_{2 m}(x, \eta)^{\lambda-m-1}(t-\eta)^{-1-Q} d \eta \leqslant \\
& \leqslant c M_{\lambda}(f) \int_{0}^{t} \eta^{(\lambda-m-1) / 2 m}(t-\eta)^{-\frac{1}{1-Q}} d \eta \leqslant \\
& \leqslant c M M_{\lambda}(f) t^{-0+(\lambda-1) / 2 m} \int_{0}^{1} \sigma^{(\lambda-m-1) / 2 m}(1-\sigma)^{-\frac{1}{2}-Q} d \sigma
\end{aligned}
$$

se $t \geqslant t_{2 m}(x)$, invece, si ha:

$$
\begin{aligned}
|h(x, t)| & =c\left|\int_{0}^{t_{2} m(x)} f_{\alpha}(x, \eta)(t-\eta)^{-\frac{1}{-}-\varrho} d \eta\right| \leqslant \\
& \leqslant c M_{\lambda}(f) \int_{0}^{t_{2 m}(x)} \eta^{(\lambda-m-1) / 2 m} \cdot(t-\eta)^{-1}-e d \eta \leqslant \\
& \leqslant c M_{\lambda}(f) t_{2 m}(x)^{\beta} \int_{0}^{t} \eta^{-\beta+\lambda-\lambda-m-1) / 2 m} \cdot(t-\eta)^{-\frac{1}{2}-e} d \eta \leqslant \\
& \leqslant c M_{\lambda}(f) t_{2 m}(x)^{\beta \cdot} \cdot t^{-Q-\beta+(\lambda-1) / 2 m} \int_{0}^{1} \sigma^{-\beta+(\lambda-m-1) / 2 m} \cdot(1-\sigma)^{-1-e} d \sigma .
\end{aligned}
$$

Dalla (3.12), così provata, consegue che:

$$
\left(\int_{G} d x \int_{-\infty}^{+\infty} h^{2}(x, t) d t\right)^{\frac{1}{2}} \leqslant c \varepsilon^{\lambda-\frac{1}{2}} M_{\lambda}(f)
$$

Infatti, per la (3.12), si ha:

$$
\begin{aligned}
\int_{-\infty}^{+\infty} h^{2}(x, l) d t & \leqslant c M_{\lambda}^{2}(f)\left\{\int_{0}^{t_{2} m(x)} t^{-2 \varrho+(\lambda-1) / m} d t+t_{2 m}(x)^{2 \beta} \int_{t_{2 m}(x)}^{+\infty} t^{-2 Q-2 \beta+(\lambda-1)) / m} d t\right\} \leqslant \\
& \leqslant c M_{\lambda}^{2}(f)\left\{t_{2 m}(x)^{-2 \varrho+(m+\lambda-1) / m}+t_{2 m}(x)^{2 \beta} t_{2 m}(x)^{-2 Q-2 \beta+(m+\lambda-1) / m}\right\} \leqslant \\
& \leqslant c M_{\lambda}^{2}(f) t_{2 m}(x)^{-2 Q+(m+\lambda-1) / m}=c M_{\lambda}^{2}(f) t_{2 m}(x)^{(2 \lambda-1) / 2 m} \leqslant \\
& \leqslant c M_{\lambda}^{2}(f) \varepsilon^{2 \lambda-1} \quad \forall x \in G
\end{aligned}
$$

da cui si deduce immediatamente la (3.13).

$$
3 \text { - Annali di Matematica }
$$


La funzione $h$ verifica anche la proprietà che, qui di seguito, enunciamo. Posto:

$$
D^{\left(\frac{1}{8}-\varrho\right)} h(x, t)=\frac{1}{\Gamma\left(\frac{1}{2}+\varrho\right)} \frac{d}{d t} \int_{0}^{t} h(x, \eta)(t-\eta)^{-\frac{1}{2}+\varrho} d \eta \quad((x, t) \in G \times \boldsymbol{R})\left({ }^{17}\right)
$$

risulta:

$$
f^{*}(x, t)=D^{\left(\frac{1}{2}-g\right)} h(x, t) \quad(t \in \boldsymbol{R}) .
$$

In effetti, per $t>0$, da (3.11) e (3.14) si ha:

$$
\begin{aligned}
D^{\left(\frac{1}{2}-\varrho\right)} h=\frac{1}{\Gamma\left(\frac{1}{2}+\varrho\right) \Gamma\left(\frac{1}{2}-\varrho\right)} & \frac{d}{d t} \int_{0}^{t}(t-\eta)^{-\frac{1}{2}+\varrho} d \eta \int_{0}^{\eta} f^{*}(x, \sigma)(\eta-\sigma)^{-\frac{1}{2}-\varrho} d \sigma= \\
& =\frac{1}{\Gamma\left(\frac{1}{2}+\varrho\right) \Gamma\left(\frac{1}{2}-\varrho\right)} \frac{d}{d t} \int_{0}^{t} f^{*}(x, \sigma) d \sigma \int_{\sigma}^{t}(t-\eta)^{-\frac{1}{2}+\varrho}(\eta-\sigma)^{-1-\varrho} d \eta
\end{aligned}
$$

poichè:

$$
\int_{\sigma}^{t}(t-\eta)^{-\frac{1}{2}+\varrho}(\eta-\sigma)^{-\frac{1}{2}-Q} d \eta=\int_{0}^{1} \theta^{-\frac{1}{2}+e}(\mathbf{1}-\theta)^{-\frac{1}{2}-\varrho} d \theta=\Gamma\left(\frac{1}{2}+\varrho\right) \Gamma\left(\frac{1}{2}-\varrho\right)
$$

si conclude che sussiste la (3.15).

Premesso tutto ciò, se $\tau \in \mathfrak{D}(\Omega)$ e $P \tau$ è un arbitrario prolungamento di $\tau$ tale che $P_{\tau} \in \mathfrak{D}(G \times \boldsymbol{R})$, tenendo presenti (3.13), (3.14), (3.15) e per proprietà della trasformata di FourIen ( $\left.{ }^{18}\right)$, si ha:

$$
\begin{aligned}
& \iint_{\Omega} f_{\alpha} \cdot \tilde{\tau} d x d t|=| \int_{G} d x \int_{0}^{t_{m} m(x)} f^{*} \cdot \tau d x d t|=| \int_{G} d x \int_{-\infty}^{+\infty} f^{*} \cdot P \tau d t|=| \int_{G} d x \int_{-\infty}^{+\infty} D^{\left(\frac{3}{2}-\rho\right)} h \cdot P \tau d t \mid \leqslant \\
& \leqslant c \int_{\alpha} d x \int_{-\infty}^{+\infty}|\sigma|^{z-Q}|\mathfrak{F}(h) \| \mathcal{F}(P \tau)| d \sigma \leqslant c\left(\int_{\sigma} d x \int_{-\infty}^{+\infty}|\mathfrak{F}(h)|^{2} d \sigma\right)^{\frac{1}{1}} \cdot\left(\int_{\sigma} d x \int_{-\infty}^{+\infty}|\sigma|^{1-2 e}|\mathfrak{F}(P \tau)|^{2} d \sigma\right)^{\frac{1}{2}} \leqslant \\
& \leqslant c\left(\int_{\sigma} d x \int_{-\infty}^{+\infty}|h|^{2} d t\right)^{\frac{1}{2}} \cdot\left(\int_{\sigma} d x \int_{-\infty}^{+\infty}|\sigma|^{1-2 Q}|\mathfrak{F}(P \tau)|^{2} d \sigma\right)^{\frac{1}{2}} \leqslant c \varepsilon^{\lambda-\frac{1}{2}} M_{\lambda}(f) \cdot\left(\int_{\theta} d x \int_{-\infty}^{+\infty}|\sigma|^{1-2 Q}|\mathfrak{F}(P \tau)|^{2} d \sigma\right)^{\frac{1}{2}} .
\end{aligned}
$$

(17) $D^{\left.\frac{1}{2}-Q\right)}(h)$ denota la derivata di ordine frazionario $\frac{1}{2}-\varrho$ di $h$ (la funzione $x^{-\frac{1}{2}+\varphi}$ si intende prolungata per $x \leqslant 0$, ponendola eguale a zero). Cfr. loc. cit. nella nota precedente.

${ }^{(18)}$ Cfr. nota $\left({ }^{6}\right)$. 
Poichè, d'altra parte:

$$
\begin{aligned}
\left.\int_{-\infty}^{+\infty}\left|\sigma^{1-2 e}\right| \mathcal{F}(P \tau)\right|^{2} d \sigma & =\int_{|\sigma|<1}|\sigma|^{1-2 e}|\mathcal{F}(P \tau)|^{2} d \sigma+\int_{|\sigma| \geqslant 1}|\sigma|^{1-\varsigma \varrho}|\mathcal{F}(P \tau)|^{2} d \sigma \leqslant \\
& \leqslant \int_{-\infty}^{+\infty}|\mathcal{F}(P \tau)|^{2} d \sigma+\int_{-\infty}^{+\infty}|\sigma||\mathcal{F}(P \tau)|^{2} d \sigma
\end{aligned}
$$

si conclude che:

$$
\left|\iint_{\Omega, s} f_{\alpha} \cdot \bar{\tau} d x d t\right| \leqslant c \varepsilon^{\lambda-\frac{1}{2}} M_{\lambda}(f) \cdot\|P \tau, G \times \boldsymbol{R}\|_{m, \frac{1}{2}}
$$

per ogni $P \tau \in \mathscr{D}(G \times \boldsymbol{R})$ ed, in conseguenza, per ogni $P \tau \in{ }^{0} W_{2}^{m, \frac{1}{2}}(G \times \boldsymbol{R})$.

Consegue, immediatamente, la seconda delle (3.10) e, così, il lemma è dimostrato.

Dimostriamo, ora, il teorema seguente relativo al problema (3.8), nel caso omogeneo.

Teorema 3.2. - Se $a_{p, q} \in C^{0}(\bar{\Omega})(|p|,|q| \leqslant m)$, se $\partial G$ \& infinitamente differenziabite e se sono verificate le ipotesi (3.3), (3.4), il problema (3.8) con $\varphi_{j}=0(j=1, \ldots, m)$, $\psi=0$ ammette un'unica soluzione

$$
u \in_{S}^{0} W_{2}^{m, s}(\Omega) \cap H^{m, 0, \mu t}(\Omega) \cap C_{x, t}^{m-1+\lambda, \lambda / 2 m}(\bar{\Omega})
$$

per la quale sussiste la formula di maggiorazione:

$$
|u, \bar{\Omega}|_{m-1,0}^{(\lambda, 2 m)}+\|u, \Omega\|_{m, \frac{1}{2}}+[u, \Omega]_{m, 0}^{(\mu)} \leqslant c M I_{\lambda}(f)
$$

ove e è una costante dipendente dai massimi moduli dei coefficienti $a_{p, q}(|p| \cdot|q| \leqslant m)$, dai moduli di continuità di $a_{\boldsymbol{p}, q}(|p|,|q|=m)$, dalla costante di parabolicità $\delta$ e da $\Omega$, mentre $\mu=n+2 m+2 \lambda-2$.

Dimostrazione. - In conseguenza del Lemma 3.1 e per un teorema di J. KaDLEC $\left({ }^{29}\right)$, il problema in considerazione ammette una unica soluzione $u \in{ }_{s}^{0} W_{2}^{m, \frac{k}{2}}(\Omega)$ e per essa sussiste la maggiorazione:

$$
\left\|u_{2}, \Omega\right\|_{m, \frac{1}{2}} \leqslant 0\left\{\sum_{|\alpha| \leqslant m} \sup _{\langle x, t) \in \Omega-\Omega_{\varepsilon}}\left|f_{\alpha}(x, t)\right|+\varepsilon^{\lambda-\frac{1}{2}} M_{\lambda}(t)\right\}
$$

da cui, ricordando la (1.1), si deduce:

$$
\|u, \Omega\|_{m, \frac{1}{2}} \leqslant c\left\{\sum_{|x| \leqslant m} \sup _{(x, t) \in \Omega-\Omega_{\varepsilon}} \mid f_{\alpha}(x, t)+\varepsilon^{\lambda-\frac{1}{2}} M_{\lambda}(f)\right\} .
$$

(19) Cfr. [8], Teor. 4.10. 
Poniamo:

Consideriamo l'equazione:

$$
\begin{aligned}
& \chi^{(\varepsilon)}(x, t)=\left\{\begin{array}{lll}
1 & \text { se }(x, t) \in \bar{\Omega}-\Omega_{\varepsilon} & \\
0 & \text { se }(x, t) \in \Omega_{\varepsilon / 2} & \left(\chi^{(\varepsilon)}(x, t) \mid \leqslant 1 \text { in } \bar{\Omega}\right.
\end{array}\right. \\
& f_{\alpha}^{(\varepsilon)}=\chi^{(\varepsilon)} f_{\alpha}(|\alpha| \leqslant m) .
\end{aligned}
$$

$$
B(u, \tau)=F\left(!^{(e)}, \tau\right)
$$

nonchè l'equazione:

$$
B_{h}(u, \tau)=F\left(f_{n}^{(e)}, \tau\right)
$$

ove $B_{h}$ sono forme bilineari analoghe a $B$ i cui coefficienti sono polinomi convergenti uniformemente verso gli omologhi coefficienti di $B$ mentre $f_{h}^{(8)}$ è un sistema di funzioni di classe $C^{\infty}(\bar{\Omega})$, nulle in un intorno della superficie laterale e della base inferiore del cilindro $\bar{\Omega}$, convergenti uniformemente in $\bar{\Omega}$ verso le omologhe componenti del sistema $f^{(\varepsilon)}$. Si procede, quindi, come nella dimostrazione del nostro analogo teorema relativo al caso in cui le funzioni $f_{\alpha}(|\alpha| \leqslant m)$ possono diventare infinitamente grandi solo sulla superficie laterale di $\bar{L}\left({ }^{20}\right)$.

In sostanza, se $u^{(8)}, u_{h}^{(\varepsilon)}$ sono, rispettivamente, le soluzioni di classe ${ }_{s}^{0} W_{2}^{m, \frac{t}{2}}(\Omega)$ delle equazioni (3.18), (3.19), si prova, utilizzando (3.17), che:

$$
\left.\begin{array}{c}
\lim _{\varepsilon \rightarrow 0}\left\|u-u^{(\varepsilon)}, \Omega\right\|_{m, \frac{1}{3}}=0 \\
\left.\lim _{h \rightarrow \infty} \| u^{(\varepsilon)}-u_{h}^{(\varepsilon)}, G \times\right] 0, T^{\prime}\left[\|_{m, 0}=0\right. \\
\|u, \Omega\|_{m, \frac{1}{2}} \leqslant c M_{\lambda}(f) .
\end{array} \quad \forall T^{\prime} \in\right] 0, T[
$$

Da ciò e dal fatto che $u u_{h}^{(6)}$ coincide quasi ovunque con una soluzione di classe $C^{\infty}(\bar{\Omega})$ del problema per la quale sussiste la maggiorazione:

$$
\mid u_{h}^{(\varepsilon)}, \bar{\Omega}\left[\left.\right|_{m-1,0} ^{(\lambda)}+\left[u_{h}^{(\varepsilon)}, \Omega\right]_{m, 0}^{(\mu)} \leqslant c M_{\lambda}\left(f_{h}^{(\ell)}\right)\right.
$$

consegue la tesi. Per i dettagli rinviamo alla dimostrazione citata.

Un risultato, analogo al precedente, relativo al problema non omogeneo si ottiene utilizzando il teorema precedente nonchè una funzione ausiliaria che verifica assegnate condizioni al contorno la cui esistenza è provata nel lemma seguente.

Lennus 3.3 - Nelle ipotesi (3.2), (3.3), (3.5), (3.6), (3.7), posto:

$$
\mathcal{L}=\sum_{|p|,|q| \leqslant m} D^{p}\left(a_{p, q} D\right)^{q}+\frac{\partial}{\partial t}
$$

${ }^{(20)}$ Cfr. [2], Lemmi 6.1, 6.2 e Teor. 6.3. 
esiste una funzione

$$
z \in C_{x, t}^{m-1+\lambda, \lambda / 2 m}(\bar{\Omega}) \cap C_{x, t}^{2 m, 1}(\Omega) \cap{ }_{R} W_{2}^{m, \frac{1}{2}}(\Omega) \cap H^{m, 0, \mu}(\Omega)
$$

verificante le condizioni al contorno:

$$
\begin{cases}\frac{d^{j-\mathrm{t} z}}{d \nu^{j-1}}\left(x^{\prime}, t\right)=\varphi_{j}\left(x^{\prime}, t\right) & \left(j=1, \ldots, m ;\left(x^{\prime}, t\right) \in \Omega^{\prime}\right) \\ \approx(x, 0)=\psi(x) & (x \in \bar{G})\end{cases}
$$

nonchè le disuguaglianze:

$$
\left\{\begin{array}{l}
|z, \bar{\Omega}|_{m-1,0}^{(\lambda, 2 m)}+\|z, \Omega\|_{m, \frac{1}{2}}+[z, \Omega]_{m, 0}^{(\mu)} \leqslant c\left(\left|\Phi, \bar{\Omega}^{\prime}\right|_{m-1,0}^{(\lambda)}+|\psi, \bar{G}|_{m-1}^{(\lambda)}\right) \\
|\mathcal{L}(z)| \leqslant c d_{2, m}(x, t)^{-m-1+\lambda}\left(\left|\Phi, \bar{\Omega}^{\prime}\right|_{m-1,0}^{(\lambda)}+|\psi, \bar{G}|_{m-1}^{(\lambda)}\right)
\end{array}\right.
$$

ove

$$
\Phi,\left.\bar{\Omega}^{\prime}\right|_{m-1,0} ^{(2)}=\sum_{j=1}^{m}\left|\varphi_{j}, \partial G \times[0, T]\right|_{m-j, 0}^{(\lambda, 2 m)}
$$

$\mu=n+2 m+2 \lambda-2$, con $e$ costante positiva dipendente da $\mathcal{L}, \lambda, \Omega$.

DTmostrazione. - Prolunghiamo, innanzitutto, l'operatore $\mathcal{L}$ in tutto lo strato $\boldsymbol{R}^{n} \times[0, T]$ in modo che siano verificate per il prolungamento, che denoteremo ancora con $\mathcal{L}$, le ipotesi del Lemma $\left.2.1{ }^{21}\right)$. Prolunghiamo, inoltre, $\psi$ in una funzione, che denoteremo con lo stesso simbolo, appartenente a $C^{m-1+\lambda}\left(\boldsymbol{R}^{n}\right)$, in modo che si abbia:

$$
\left.\left|\psi ; \boldsymbol{R}^{n}\right|_{m-1}^{(\lambda)} \leqslant e|\psi, \bar{G}|_{m-1}^{(\lambda)}{ }^{(22)}\right)
$$

Consideriamo la funzione $w$, fornita dal Lemma 2.1 , relativa ai suddetti prolungamenti di $\mathcal{L}$ e $\psi$.

Posto:

$$
\varphi_{j}^{\prime}\left(x^{\prime}, t\right)=\varphi_{j}\left(x^{\prime}, t\right)-\frac{d^{j-1} w}{d v^{j-1}}\left(x^{\prime}, t\right) \quad\left(j=1, \ldots, m ;\left(x^{\prime}, t\right) \in \Omega^{\prime}\right)
$$

da (3.7) consegue:

$$
\varphi_{j}^{\prime}\left(x^{\prime}, 0\right)=\varphi_{j}\left(x^{\prime}, 0\right)-\frac{d^{j-1} \psi}{d w^{j-1}}\left(x^{\prime}\right)=0 \quad\left(j=1, \ldots, m ; x^{\prime} \in \partial G\right)
$$

È possibile, allora, ricorrendo a rappresentazioni parametriche locali di $\partial G$ ed

(21) Cfr. [6], p. 81.

(22) Cfr. [11], Lemma 4.1. 
applicando il Lemma 2.2 , costruire una funzione:

$$
v \in C_{x, t}^{m-1+\lambda, \lambda / 2 m}(\bar{\Omega}) \cap C_{x, t}^{2 m, 1}(\Omega) \cap_{R} W_{2}^{m, \frac{1}{2}}(\Omega) \cap H^{m, 0, \mu}(\Omega)
$$

tale che:

$$
\begin{cases}\frac{d^{j-1} v}{d v^{j-1}}\left(x^{\prime}, t\right)=\varphi_{j}\left(x^{\prime}, t\right) & \left(j=1, \ldots, m ;\left(x^{\prime}, t\right) \in \Omega^{\prime}\right) \\ v(x, 0)=0 & (x \in \bar{G}) \\ |\mathcal{L}(v)| \leqslant c d_{2 m}(x, t)^{-m-1+\lambda}\left|\Phi^{\prime}, \bar{\Omega}^{\prime}\right|_{m-1,0}^{(\lambda)} & \\ v v,\left.\bar{\Omega}\right|_{m-1,0} ^{(\lambda, 2 m)}+\|v, \Omega\|_{m,}+[v, \Omega]_{m, 0}^{(\mu)} \leqslant o\left|\Phi^{\prime}, \bar{\Omega}^{\prime}\right|_{m-1,0}^{(\lambda)}\end{cases}
$$

ove $\left|\Phi^{\prime}, \bar{\Omega}^{\prime}\right|_{m-1,0}^{(\lambda)}$ si ottiene formalmente da $\mid \Phi, \bar{\Omega}^{\prime}{ }^{\prime(\lambda)-1,0}$ sostituendo $\varphi_{j}$ con $\varphi_{j}^{\prime}\left({ }^{23}\right)$

La funzione $z=v+w$ verifica $(3.20),(3.21)$, (3.22).

Possiamo, ora, stabilire il teorema seguente che è lo scopo principale di questo lavoro.

TEoREma 3.4. - Nelle ipotesi (3.2), .., (3.7), il problema (3.8) ammette un'unica soluzione:

$$
u \in \in_{R} W_{2}^{m, \frac{1}{1}}(\Omega) \cap H^{m, 0, \mu}(\Omega) \cap C_{x, t}^{m-1+\hat{\lambda}, \lambda / 2 m}(\bar{\Omega})
$$

per la qquale sussiste la formula di maggiorazione:

$$
|u, \bar{\Omega}|_{m-1,0}^{(\lambda, 2 m)}+\|u, \Omega\|_{m, \frac{\alpha}{2}}+[u, \Omega]_{m, 0}^{(\mu)} \leqslant c\left(M_{\lambda}(f)+i \Phi,\left.\bar{\Omega}_{m}^{\prime(\lambda)}\right|_{m-1,0} ^{(\lambda)}+|\psi, \bar{G}|_{m-1}^{(\lambda)}\right)
$$

ove:

$$
\left|\Phi_{j} \bar{\Omega}^{\prime}\right|_{m-1,0}^{(\lambda)}=\sum_{j=1}^{m}\left|\varphi_{j}, \partial G \times[0, T]\right|_{m-j, 0}^{(\lambda, 2 m)}
$$

$\mu=n+2 m+2 \lambda-2$ o $\circ$ è una costante positiva dipendente solo dai coefficientì dell'equazione e dall'insieme $\Omega$.

Dimostrazione. - La tesi si deduce dal Teorema 3.2 e dal Lemma 3.3 con un semplice ragionamento $\left({ }^{24}\right)$ del quale, comunque, per comodità del lettore, daremo un breve cenno.

Se $z$ ed $\mathfrak{L}$ sono, rispettivamente, la funzione e l'operatore cui si fa riferimento nel Lemma 3.3, posto:

$$
g=\left\{\begin{array}{ll}
f_{\alpha} & \text { se } \alpha \neq 0 \\
f_{0}-\mathcal{L}(z) & \text { se } \alpha=0
\end{array} \quad g=\left(g_{\alpha}\right)_{|\alpha| \leqslant m}\right.
$$

${ }^{(23)}$ Cfr. [9], dimostrazione del Teor. IV

(24) Cfr. [3], dimostrazione del Teor. 3.3. 
da (3.22) consegue:

$$
M_{\lambda}(g) \leqslant M_{\lambda}(f)+e\left(\left|\Phi, \bar{Q}^{\prime}\right|_{m-1,0}^{(\lambda)}+\mid \psi, \bar{G}_{i m-1}^{(\lambda)}\right)
$$

Pertanto, esiste $u_{0} \in{ }_{S} W_{2}^{m, \frac{1}{2}}(\Omega) \cap H^{m_{i}, 0, \mu}(\Omega) \cap C_{x, t}^{m-1+\lambda_{3} \lambda / 2 m}(\bar{\Omega})$ soluzione del problema:

$$
\begin{cases}B(u, \tau)=\mathbb{F}(g, \tau) & \forall \tau \in \mathcal{D}(\Omega) \\ \frac{d^{j-1} u}{d y^{j-1}}\left(x^{\prime}, t\right)=0 & \left(j=1, \ldots, m ;\left(x^{\prime}, t\right) \in \Omega^{\prime}\right) \\ u(x, 0)=0 & (x \in \bar{G}) .\end{cases}
$$

Allora la funzione $u=u_{0}+z$ è soluzione del problema (3.8) e verifica (3.23) in conseguenza della (3.24), della disuguaglianza (3.16) (con $f$ sostituito da $g$ ) verificata da $u_{0}$ e della disuguaglianza (3.22) verificata da $z$.

L'unicità consegue dal Teorema 3.2 .

\section{BIBLIOGRAFIA}

[1] S. AGMon, Maximum theorem for solutions of higher order elliptic equations, Bull. Am. Math. Soc., 66 (1960), pp. 77-80.

[2] G. ARNEse, Teorema del massimo modulo per il problema omogeneo di Diriohlet per una equazione parabolica $d i$ ordine $2 m$ in $n$ variabili, Ricerche di Matematica, 17 (1968), pp. 21-63.

[3] G. AnNese, Teorema di esistenza ed unicità per il problema di Dirichlet per un'equazione parabolica di ordine $2 m$ in $n$ variabili, Ed, Liguori, Napoli, 1968.

[4] G. AnNese, Su alcune proprietà dell'integrale di Poisson relativo ad un'equazione parabolica di ordine $2 m$, a coefficienti non costanti, Annali Mat. pura appl., questo stesso volume.

[5] G. Arnese, Maggiorazioni in $I^{p}$ dei potenziali relativi all'equazioni del calore, Ricerche di Matematica, 13 (1964), pp. 147-191.

[6] A. Friedman, Partial differential equations of parabolic type, Prentice Hall, Englewood (N.J.), 1964.

[7] I. M. Guelfand, G. E. Chulov, Les aistributions, Dunod, Paris, 1962.

[8] J. KADLEC, Soluzione del primo problema ai limiti per una generalizazione dell'equazione del calore in classi di funzioni con derivate frazionarie rispetto al tempo, Cecoslovak Mat. J., 16 (1966), pp. 91-113.

[9] C. MiRanda, Teorema del massimo modulo e teorema di esistenza ed unicità per il problema di Dirichlet relativo alle equazioni ellittiche in due variabili, Annali Mat. pura appl., s. IV, 46 (1958), pp. 265-312.

[10] L. Schwartz, Théorie des distributions, Hermann, Paris, 1966.

[11] O. A. Solonnikov, Problemi al contorno per sistemi di equazioni differenziali lineari parabolici di tipo generale, Trudy Mat. Inst. V. A. Steklov, 83 (1965). 